\title{
BioMedicine
}

Volume 11 | Issue 3

Article 1

2021

\section{Nosocomial COVID-19 infection in patients with nasal trauma during coronavirus pandemic}

Follow this and additional works at: https://www.biomedicinej.com/biomedicine

Part of the Life Sciences Commons, and the Medical Sciences Commons

(c) () ()

This work is licensed under a Creative Commons Attribution 4.0 License.

\section{Recommended Citation}

Erfanian, Reza; Manavi, Mahdokht; Sohrabpour, Saeed; Irani, Shirin; and Firouzifar, Mohammadreza (2021) "Nosocomial COVID-19 infection in patients with nasal trauma during coronavirus pandemic,"

BioMedicine: Vol. 11 : Iss. 3 , Article 1.

DOI: $10.37796 / 2211-8039.1199$

This Short Communications is brought to you for free and open access by BioMedicine. It has been accepted for inclusion in BioMedicine by an authorized editor of BioMedicine. 
Nosocomial COVID-19 infection in patients with nasal trauma during coronavirus pandemic

\section{Cover Page Footnote}

None. 


\title{
Nosocomial COVID-19 infection in patients with nasal trauma during coronavirus pandemic
}

\author{
Reza Erfanian ${ }^{\mathrm{a}}$, Mahdokht Manavi ${ }^{\mathrm{b}}$, Saeed Sohrabpour ${ }^{\mathrm{a}}$, \\ Shirin Irani ${ }^{a}$, Mohammadreza Firouzifar ${ }^{a, *}$ \\ ${ }^{a}$ Assistant Professor, Otorhinolaryngology Research Center, Tehran University of Medical Sciences, Tehran, Iran \\ ${ }^{\mathrm{b}}$ Rhinology Fellowship Resident, Otorhinolaryngology Research Center, Tehran University of Medical Sciences, Tehran, Iran
}

\begin{abstract}
Objectives: Considering the reduction of patients' attendance in hospitals, including patients with nose fracture, in fear of COVID-19 transmission, this study aims to investigate the risk of nosocomial COVID-19 transmission in patients with nasal trauma seeking medical care in hospital.

Methods: Patients with nasal trauma referred to our specialized ENT referral center were evaluated, treated, and followed, up to two weeks. After discharge, COVID-19 symptoms were questioned by telephone, and additional assessments were requested for suspected patients.

Results: Out of 108 patients with possible nasal fracture, 27 didn't need nasal reduction after physical examination while 81 required nasal bone reduction. Only one of the patients contracted the virus in the 2-week follow-up period, and the difference between the two groups was not statistically significant.

Conclusion: Patients in need of emergency medical services -like nasal trauma-in outpatient and short-term inpatient settings should not avoid going to the hospitals. This is provided when both medical staff and patients follow the health protocols.
\end{abstract}

Keywords: Coronavirus, COVID-19, Cross Infection, Infection Control, SARS-CoV-2, Nose trauma

\section{Introduction}

$\mathrm{T}$ he novel coronavirus, also known as severe acute respiratory syndrome coronavirus 2 (SARS-CoV-2), causes coronavirus disease 2019 (COVID-19) and its complications, which pose a unique challenge for disease control and prevention in medical centers [1, 2]. The disease causes a broad spectrum of symptoms, from asymptomatic infection to mild upper respiratory tract disease, severe viral pneumonia with respiratory failure, and even death [3]. Although many infected people have no symptoms, symptomatic patients experience fever, dry cough, fatigue, lethargy, and shortness of breath [4]. COVID-19 is highly contagious, the incubation period is reported to be 5 to 7 to even 14 days, and it is transmitted through aerosols and close contact with an infected person [5-7]. In fact, this is an airborne disease that can be transmitted from an asymptomatic or symptomatic person to others [8]. Also, the virus can be transmitted from surfaces for up to 3 days [6].

Nosocomial infections are infections transmitted to a patient that is hospitalized for other reasons [1]. There is insufficient data on the chances of coronavirus transmission in hospitals [9]. However, nonCOVID-19 patients in need of health services refuse to go to hospitals even in emergencies during the pandemic in fear of contracting COVID-19. This significant reduction in patients' visits causes progressive morbidity and mortality [10].

Treatment of nasal fractures is an emergency, especially in the presence of septal hematoma and/ or active bleeding. In addition, nasal bone fractures with extensive displacement can cause a nonreversible deformity if the person does not seek

Received 23 January 2021; revised 17 February 2021; accepted 22 March 2021.

Available online 1 September 2021.

* Corresponding author at: Assistant Professor, Otorhinolaryngology Research Center, Tehran University of Medical Sciences, Amir Alam Hospital, Iran. E-mail address: mrfirouzifar@sina.tums.ac.ir (M. Firouzifar). 
medical care in time [11, 12]. The procedure is possible in outpatient or 1-day inpatient settings [11]. Definite nosocomial infection in these patients is defined as the presence of symptoms fourteen days or more after seeking medical care in a hospital, and probable nosocomial infection is the onset of symptoms within seven days of hospital admission with a proven contact with a COVID-19 patient in the hospital $[1,2,13]$. Due to the lack of sufficient information, this study aims to investigate the risk of nosocomial COVID-19 in patients with nasal trauma seeking medical care in the hospital.

\section{Materials and methods}

Institutional Ethics review board approved this study protocol. In this prospective study, patients referred to the Marvasti Hospital, which is a specialized ENT referral center, from June to August 2020 were evaluated, treated, and followed up to two weeks after their first visit. Patients with previous COVID-19 were excluded from the study. Patients with suggestive symptoms (e.g. fever, dyspnea, and cough) were recorded. We also used standard screening protocols (COVID Ab, PCR or chest CT scan) to reveal the infection status of the patients. Asymptomatic patients with COVID infection were excluded from the study. There were no age or gender limitations.

Patients with nasal fractures underwent nasal bone reduction. Patients over 14 years of age were treated in an outpatient setting, while the patients under 14 years of age and adult patients who could not tolerate the procedure under local anesthesia needed a day of hospitalization to treat the fracture under general anesthesia. During the procedure, all the medical staff used personal protective equipment, including masks and disposable gloves and performed frequent washing and hand sterilization in accordance with the institutional health protocols.

Patients were contacted after their discharge to check for clinical COVID-19 symptoms between 5 and 14 days after their procedure. Fever, cough, weakness, shortness of breath, and olfactory dysfunction were asked of all patients, and they could also report any other symptoms to the physician. Coronavirus PCR or chest CT scan was requested in patients who were suspicious of COVID-19 infection.

\section{Results}

One hundred eight patients including 30 females and 78 males with a mean age of 36 years (range, 6 to 77 years) referred to the Nose fracture emergency department of Marvasti Hospital, Tehran, Iran from June to August 2020.

Eighty-one of these patients - 19 women and 62 men - needed fracture reduction (group A), and 27 patients - 11 women and 16 men - did not need reduction after the physical examination (group B). The gender difference between the groups was not significant.

Seven patients under 14 years of age were transferred to the operating room due to intolerance to the outpatient procedure. In other patients, the nasal bone reduction was performed on an outpatient basis after infra-orbital and supratrochlear nerve blocks with $4 \%$ lidocaine and insertion of a xylocaine-soaked pledgets under the nasal bone to anesthetize the mucosa.

In the follow-up, only one out of 108 clients, who was among group A, had olfactory dysfunction ten days after his first visit. The coronavirus PCR result was positive for the patient and his wife, child, and father - with whom he had close contact - and his father needed hospitalization. Follow-up continued until the patient, and all three people in contact with him recovered. There was no statistically significant difference between groups $A$ and $B$ in terms of nosocomial COVID-19 transmission.

\section{Discussion}

Determining the risk of COVID-19 nosocomial infection is influential in macro-decisions and can help decision-makers set guidelines or advise and inform the public $[1,2,14]$. The present study showed that in outpatient and short-term hospitalizations of trauma patients, the probability of nosocomial infection is low provided that protective measures-e.g., proper ventilation in the clinic and operating room, maintaining physical distance, use of gloves, masks, and goggles by the treatment staff, and rules of mandatory masking for patients-are met. Therefore, by strictly following these measures, patients can be recommended to refer in case of emergency.

Risk factors for nosocomial infection include age over 70, immunosuppression, ICU admission, history of trauma, use of antibiotics, and catheter use [15]. As a result, the lower number of nosocomial infections in this study may be due to the outpatient management of most of the patients.

The incidence of COVID-19 nosocomial infection has been reported in studies to have a vast range. In a study in Wuhan, China, the incidence of nosocomial COVID-19 was reported to be more than $41 \%$ [16]. However, this rate was reported at the 
beginning of the pandemic, when the use of personal protective equipment was not yet standardized and common, and also, the hospitalization ward for patients with COVID-19 was not separate from other patients. These cases probably explain the reason for the high percentage of nosocomial COVID-19 infection reported in this study. In another study by Carter et al. in the UK, the rate was reported to be $12.5 \%$ [1]. Although in contrast to our study, they included the patients with longer hospital stays and patients admitted to the ICU. In contrast, in a study by Chang et al. in Hong Kong, none of the 42 patients who were admitted to the hospital developed nosocomial COVID-19 infection. In this study, personal protection, recurrent hand washing, ventilation of rooms, and isolation of patients were highly emphasized, so the adherence to restrict protocols was considered the leading cause of patient protection [17]. However, the number of included patients was less than other studies, which may impact the nosocomial infection rate.

All cases of nasal fractures can be treated electively, except in the presence of septal hematoma or active bleeding [11, 18]. Endoscopic examination of the nose should be avoided. Also, vasoconstrictor and local anesthetic agents should be used before the manipulation, for which a combination of oxymetazoline and $4 \%$ lidocaine is used [18]. Intranasal sprays should also be avoided as they have a chance of increasing secretions and spreading the virus in the environment [19].

There are some limitations in our study that should be mentioned. One of the main limitations is the lack of primary infection source identification. To determine the source, studying the genome of the virus can be helpful, which of course is not readily available in the clinic. Second limitation was that we didn't seek the incidence of infection in the treatment staff. Third limitation is that we followed our patients with phone calls and we based our post-exposure diagnosis on symptomatic patients. Our primary screening methods were also not homogenous because it was not ethical to pose extra expenses on patients to screen all of them with same exam and this also would increase the number of visits in hospital and other places like laboratories, imaging centers, and general transportation systems which would cause bios in our results. So it was wiser to limit hospital visits to one and base our preexposure diagnosis on whatever the patient could provide. Finally, our information may not be accurate for all hospitals and treatment centers because the conditions, facilities, and the number of COVID19 patients admitted can vary.

\section{Conclusion}

In this study, it was shown that if the treatment staff and patients follow the health protocols, the incidence of COVID-19 symptoms in the outpatient and short-term inpatient setting is negligible. Further high-quality studies are recommended to rectify the limitations of this study for improving guidelines and decision-making in this epidemic and subsequent possible epidemics.

\section{Acknowledgements}

None.

\section{Role of funding source}

This research did not receive any specific grant from funding agencies in the public, commercial, or not-for-profit sectors.

\section{Conflict of interest statement}

The authors have no conflict of interests.

\section{References}

[1] Carter B, Collins JT, Barlow-Pay F, Rickard F, Bruce E, Verduri A, et al. Nosocomial COVID-19 infection: examining the risk of mortality. The COPE-Nosocomial Study (COVID in Older PEople). J Hosp Infect 2020;106(2): 376-84.

[2] Rickman HM, Rampling T, Shaw K, Martinez-Garcia G, Hail L, Coen P, et al. Nosocomial transmission of COVID-19: a retrospective study of 66 hospital-acquired cases in a London teaching hospital. Clin Infect Dis 2021 Feb 16;72(4):690-3. 2020/06//. PubMed PMID: 32562422. eng.

[3] Zhou F, Yu T, Du R, Fan G, Liu Y, Liu Z, et al. Clinical course and risk factors for mortality of adult inpatients with COVID-19 in Wuhan, China: a retrospective cohort study. Lancet 2020;395(10229):1054-62.

[4] Kaul D. An overview of coronaviruses including the SARS-2 coronavirus-Molecular biology, epidemiology and clinical implications. Curr Med Res Prac 2020 Mar-Apr;10(2):54-64.

[5] Organization WHO. Clinical management of severe acute respiratory infection when Middle East respiratory syndrome coronavirus ( MERS-CoV) infection is suspected: interim guidance. World Health Organization; 2019.

[6] Van Doremalen N, Bushmaker T, Morris DH, Holbrook MG, Gamble A, Williamson BN, et al. Aerosol and surface stability of SARS-CoV-2 as compared with SARS-CoV-1. New England J Med 2020;382(16):1564-7.

[7] Lauer SA, Grantz KH, Bi Q, Jones FK, Zheng Q, Meredith HR, et al. The incubation period of coronavirus disease 2019 (COVID-19) from publicly reported confirmed cases: estimation and application. Ann Intern Med 2020; 172(9):577-82.

[8] He X, Lau EH, Wu P, Deng X, Wang J, Hao X, et al. Temporal dynamics in viral shedding and transmissibility of COVID19. Nat Med 2020;26(5):672-5.

[9] Rhee C, Baker M, Vaidya V, Tucker R, Resnick A, Morris CA, et al. Incidence of nosocomial COVID-19 in patients hospitalized at a large US academic medical center. JAMA Network Open 2020;3(9):e2020498. 
[10] Lazzerini M, Barbi E, Apicella A, Marchetti F, Cardinale F, Trobia G. Delayed access or provision of care in Italy resulting from fear of COVID-19. Lancet Child Adolesc Health 2020;4(5):e10-1.

[11] Ziccardi VB, Braidy H. Management of nasal fractures. Oral Maxillofac Surg Clin North Am 2009;21(2):203-8.

[12] Rohrich RJ, Adams Jr WP. Nasal fracture management: minimizing secondary nasal deformities. Plast Reconstr Surg 2000;106(2):266-73.

[13] Holmes S, Bhatti N, Bhandari R, Chatzopoulou D. Toward a consensus view in the management of acute facial injuries during the Covid-19 pandemic. Brit J Oral Maxillofac Surg 2020 Jun;58(5):571-6.

[14] Lu D, Wang H, Yu R, Yang H, Zhao Y. Integrated infection control strategy to minimize nosocomial infection of coronavirus disease 2019 among ENT healthcare workers. J Hosp Infect 2020;104(4):454.
[15] Li Y, Ren L, Zou J. Risk Factors and Prevention Strategies of Nosocomial Infection in Geriatric Patients. Can J Infecti Dise Med Microbiol 2019;2019.

[16] Zhou Q, Gao Y, Wang X, Liu R, Du P, Wang X, et al. Nosocomial Infections Among Patients with COVID-19, SARS and MERS: A Rapid Review and Meta-Analysis. medRxiv 2020 May;8(10):629.

[17] Cheng VC, Wong S-C, Chen JH, Yip CC, Chuang VW, Tsang $\mathrm{OT}$, et al. Escalating infection control response to the rapidly evolving epidemiology of the Coronavirus disease 2019 (COVID-19) due to SARS-CoV-2 in Hong Kong. Infect Contr Hosp Epidemiol 2020;41(5):493-8.

[18] Ghai S. Facial Trauma Management during the COVID-19 era: a primer for surgeons. Current medicine research and practice. 2020.

[19] AAoO-HaN S. Academy Supports CMS, Offers Specific Nasal Policy. 2020. 